\title{
Optimizing Water and Nitrogen Application System to Improve Nitrogen use Efficiency and Ensure Sustainable Yield of Summer Maize (Zea mays $L$.)
}

\section{Xiangfei Han ( $\nabla$ hxfei1994@126.com ) \\ Lina Dong \\ Shandong Agricultural University \\ Peng Liu \\ Shandong Agricultural University \\ Shuting Dong \\ Shandong Agricultural University \\ Jiwang Zhang \\ Shandong Agricultural University \\ Bin Zhao \\ Shandong Agricultural University \\ Baizhao Ren \\ Shandong Agricultural University}

Shandong Agricultural University https://orcid.org/0000-0003-3351-6700

\section{Research Article}

Keywords: Summer Maize, Irrigation Methods, Nitrogen Use Efficiency, Ammonia Volatilization

Posted Date: February 23rd, 2021

DOI: https://doi.org/10.21203/rs.3.rs-223117/v1

License: @) (i) This work is licensed under a Creative Commons Attribution 4.0 International License. Read Full License 


\section{Abstract}

Exploring the optimal method of water and fertilizer application and $\mathrm{N}$ application rate for summer maize is important for achieving the high water and $\mathrm{N}$ efficiency in the North China Plain. We tested that the hypothesis that optimizing water and nitrogen application system could improve nitrogen $(\mathrm{N})$ use efficiency and water productivity, and ensure sustainable yield of summer maize. The results showed that: the $216 \mathrm{~kg} \mathrm{~N} \mathrm{ha}^{-1}$ of drip irrigation (DI) and micro-sprinkling irrigation (SI) could obtain the high grain yield compared with $270 \mathrm{~kg} \mathrm{~N} \mathrm{ha}^{-1}$ of flooding irrigation (FI), which was achieved by maintaining a high 1000-grain weight and kernel number. However, the grain yield of 162 $\mathrm{kg} \mathrm{N} \mathrm{ha}^{-1}$ would be decreased significantly. Irrigation methods and $\mathrm{N}$ application rates had significant effects on the ammonia volatilization rate and ammonia volatilization accumulation of soil, $\mathrm{N}$ harvest index ( $\mathrm{NHI}), \mathrm{N}$ partial productivity and water productivity. Compared with $\mathrm{FI}, \mathrm{DI}$ and SI could reduce the ammonia volatilization rate through applying little fertilizer-N by times, and reduce the ammonia volatilization accumulation of $19.5 \%-54.9 \%$. In addition, under the same irrigation method, the $\mathrm{NHI}$ reached the maximum when the $\mathrm{N}$ application rate was $216 \mathrm{~kg} \mathrm{ha}^{-1}$. Considering comprehensively, under the condition of this experiment, $216 \mathrm{~kg} \mathrm{~N} \mathrm{ha}^{-1}$ is the best $\mathrm{N}$ application rate under DI or SI for maize.

\section{Full Text}

Due to technical limitations, full-text HTML conversion of this manuscript could not be completed. However, the latest manuscript can be downloaded and accessed as a PDF.

\section{Tables}

Table 1 Effects of different irrigation methods and nitrogen application rates on grain yields and its components of summer maize. 


\begin{tabular}{|c|c|c|c|c|c|c|}
\hline Year & $\begin{array}{l}\text { Irrigation } \\
\text { method }\end{array}$ & $\begin{array}{l}\text { Nitrogen application } \\
\text { rate } \\
\left(\mathrm{kg} \mathrm{ha}^{-1}\right)\end{array}$ & $\begin{array}{l}\text { Harvest } \\
\text { ear } \\
\left(\text { No. ha }{ }^{-1}\right)\end{array}$ & $\begin{array}{l}\text { Kernels per } \\
\text { ear } \\
\left(\text { No. ear }{ }^{-1}\right)\end{array}$ & $\begin{array}{l}\text { 1000-grain } \\
\text { weight } \\
\text { (g) }\end{array}$ & $\begin{array}{l}\text { Grain yield } \\
\left(\mathrm{kg} \mathrm{ha}^{-1}\right)\end{array}$ \\
\hline \multirow[t]{12}{*}{2017} & DI & No & 67407.4a & 497.2de & $359.6 e$ & $9036.5 d$ \\
\hline & & N162 & $68888.9 a$ & $516.7 c$ & $371.4 \mathrm{~cd}$ & $11699.9 \mathrm{~b}$ \\
\hline & & N216 & $67777.8 \mathrm{a}$ & $528.9 a b$ & 378.7ab & $12316.7 a$ \\
\hline & & N270 & $68518.5 a$ & $530.6 a$ & $381.0 a$ & $12577.5 a$ \\
\hline & SI & No & $66296.3 a$ & 496.1de & $357.3 e$ & 8799.3de \\
\hline & & N162 & $68888.9 a$ & $516.2 c$ & $373.8 \mathrm{bcd}$ & $11414.0 \mathrm{bc}$ \\
\hline & & N216 & $69629.6 a$ & $521.8 \mathrm{abc}$ & 378.2abc & $12208.1 \mathrm{a}$ \\
\hline & & N270 & 69259.3a & $527.2 \mathrm{abc}$ & $380.1 \mathrm{ab}$ & $12301.6 a$ \\
\hline & $\mathrm{Fl}$ & No & 67407.4a & $490.2 \mathrm{e}$ & $356.5 \mathrm{e}$ & $8554.7 \mathrm{e}$ \\
\hline & & N162 & $68888.9 a$ & $504.7 d$ & $367.7 d$ & $10985.3 c$ \\
\hline & & N216 & $68888.9 a$ & $518.8 \mathrm{bc}$ & $373.7 \mathrm{bcd}$ & $11467.5 b$ \\
\hline & & N270 & $68888.9 a$ & $522.1 \mathrm{abc}$ & 378.8ab & $12160.7 a$ \\
\hline \multirow[t]{12}{*}{2018} & DI & NO & 65185.2ab & $466.7 f$ & $340.0 \mathrm{~b}$ & $8756.9 d$ \\
\hline & & N162 & $66666.7 a$ & 493.9def & $359.1 \mathrm{a}$ & 10167.5b \\
\hline & & N216 & 64814.8ab & 536.7ab & $366.9 a$ & 11166.1a \\
\hline & & N270 & 65185.2ab & $550.1 \mathrm{a}$ & $368.2 a$ & $11529.5 a$ \\
\hline & SI & NO & 64074.1ab & $504.2 \mathrm{cde}$ & $334.6 \mathrm{~b}$ & $9319.3 c$ \\
\hline & & N162 & 64444.4ab & $519.4 \mathrm{bcd}$ & $361.0 a$ & $10370.0 \mathrm{~b}$ \\
\hline & & N216 & $65185.2 \mathrm{ab}$ & $543.1 \mathrm{ab}$ & $366.5 a$ & 11091.7a \\
\hline & & N270 & 64074.0ab & $548.4 a$ & $368.2 a$ & 11495.7a \\
\hline & $\mathrm{FI}$ & NO & $62962.9 b$ & $479.1 \mathrm{ef}$ & $338.7 \mathrm{~b}$ & $8170.6 \mathrm{e}$ \\
\hline & & N162 & 63333.3b & $502.2 \mathrm{de}$ & $358.3 a$ & $9091.8 \mathrm{~cd}$ \\
\hline & & N216 & $63703.7 b$ & $518.7 \mathrm{bcd}$ & $362.3 a$ & $10575.6 b$ \\
\hline & & N270 & 64814.8ab & 531.3abc & $364.4 a$ & $11340.7 a$ \\
\hline \multicolumn{7}{|c|}{ ANOVA } \\
\hline I & & & 0.421 & 0.002 & 0.111 & $<0.001$ \\
\hline$N$ & & & 0.029 & $<0.001$ & $<0.001$ & $<0.001$ \\
\hline $1 \times N$ & & & 0.546 & 0.070 & 0.434 & 0.286 \\
\hline
\end{tabular}

Note: Different letters in a row are significant differences among treatments in each year $(\mathrm{P}<0.05)$. I: Irrigation methods, $\mathrm{N}$ : N application rates. DI: drip irrigation, SI: micro-sprinkler irrigation, Fl: flooding irrigation. $\mathrm{NO}: 0 \mathrm{~kg} \mathrm{~N} \mathrm{ha-1}$, 
N162: $162 \mathrm{~kg} \mathrm{~N} \mathrm{ha}^{-1}, \mathrm{~N} 216: 216 \mathrm{~kg} \mathrm{~N} \mathrm{ha}^{-1}, \mathrm{~N} 270: 270 \mathrm{~kg} \mathrm{~N} \mathrm{ha}^{-1}$.

Table 2 Effects of different irrigation methods and nitrogen application rates of accumulation amounts and distribution of dry matter in pre-anthesis and post-anthesis stages of summer maize. 


\begin{tabular}{|c|c|c|c|c|c|c|c|}
\hline Year & $\begin{array}{l}\text { Irrigation } \\
\text { method }\end{array}$ & $\begin{array}{l}\text { Nitrogen } \\
\text { application } \\
\text { rate } \\
\left(\mathrm{kg} \mathrm{ha}^{-1}\right)\end{array}$ & $\begin{array}{l}\text { Biomass } \\
\text { of R6 } \\
\left(\mathrm{t} \mathrm{ha}^{-1}\right)\end{array}$ & $\begin{array}{l}\text { Pre- } \\
\text { anthesis } \\
\left(\mathrm{tha}^{-1}\right)\end{array}$ & $\begin{array}{l}\text { Post- } \\
\text { anthesis } \\
\left(\mathrm{t} \mathrm{ha}^{-1}\right)\end{array}$ & $\begin{array}{l}\text { Percentage of } \\
\text { pre-anthesis (\%) }\end{array}$ & $\begin{array}{l}\text { Percentage of } \\
\text { post-anthesis (\%) }\end{array}$ \\
\hline \multirow[t]{12}{*}{2017} & DI & NO & $17.43 \mathrm{f}$ & $6.30 \mathrm{~b}$ & $11.12 \mathrm{e}$ & 36.17 & 63.83 \\
\hline & & N162 & $20.60 \mathrm{~d}$ & $6.83 a$ & $13.78 \mathrm{c}$ & 33.17 & 66.83 \\
\hline & & N216 & $22.13 \mathrm{~b}$ & $7.03 \mathrm{a}$ & $\begin{array}{l}15.10 \\
a b\end{array}$ & 31.78 & 68.22 \\
\hline & & N270 & $22.85 \mathrm{a}$ & $7.29 a$ & $15.56 \mathrm{a}$ & 31.9 & 68.10 \\
\hline & SI & No & $16.67 \mathrm{~g}$ & $6.07 \mathrm{~b}$ & $10.61 \mathrm{e}$ & 36.36 & 63.64 \\
\hline & & N162 & $20.90 \mathrm{~cd}$ & $6.88 \mathrm{a}$ & $\begin{array}{l}14.03 \\
b c\end{array}$ & 32.96 & 67.04 \\
\hline & & N216 & $22.46 \mathrm{ab}$ & $6.98 \mathrm{a}$ & $15.48 \mathrm{a}$ & 31.1 & 68.90 \\
\hline & & N270 & $22.74 a b$ & $7.01 \mathrm{a}$ & $15.74 \mathrm{a}$ & 30.8 & 69.20 \\
\hline & $\mathrm{Fl}$ & No & $16.38 \mathrm{~g}$ & $6.27 \mathrm{~b}$ & $10.12 \mathrm{e}$ & 38.26 & 61.74 \\
\hline & & N162 & $19.75 \mathrm{e}$ & $7.23 \mathrm{a}$ & $12.54 \mathrm{~d}$ & 36.53 & 63.47 \\
\hline & & N216 & $20.66 d$ & $7.16 \mathrm{a}$ & $\begin{array}{l}13.50 \\
\mathrm{~cd}\end{array}$ & 34.69 & 65.31 \\
\hline & & N270 & $21.38 c$ & $7.30 \mathrm{a}$ & $\begin{array}{l}14.07 \\
b c\end{array}$ & 34.15 & 65.85 \\
\hline \multirow[t]{12}{*}{2018} & DI & NO & $15.27 \mathrm{f}$ & $5.70 \mathrm{e}$ & $9.57 \mathrm{f}$ & 37.39 & 62.61 \\
\hline & & N162 & $20.19 b c$ & $7.44 \mathrm{ab}$ & $\begin{array}{l}12.75 \\
\mathrm{~cd}\end{array}$ & 36.85 & 63.15 \\
\hline & & N216 & $21.19 a b$ & $7.53 \mathrm{ab}$ & $\begin{array}{l}13.66 \\
a b\end{array}$ & 35.59 & 64.41 \\
\hline & & N270 & $21.86 \mathrm{a}$ & $7.77 \mathrm{a}$ & $14.09 \mathrm{a}$ & 35.55 & 64.45 \\
\hline & SI & NO & $14.57 \mathrm{f}$ & $5.53 \mathrm{e}$ & $9.03 \mathrm{f}$ & 37.94 & 62.06 \\
\hline & & N162 & $18.11 \mathrm{e}$ & $6.86 \mathrm{~cd}$ & $11.25 \mathrm{e}$ & 37.86 & 62.14 \\
\hline & & N216 & $19.73 \mathrm{~cd}$ & $7.12 \mathrm{bc}$ & $\begin{array}{l}12.60 \\
c d\end{array}$ & 36.1 & 63.90 \\
\hline & & N270 & $20.28 b c$ & $\begin{array}{l}7.29 \\
a b c\end{array}$ & $\begin{array}{l}12.99 \\
\mathrm{bc}\end{array}$ & 35.93 & 64.07 \\
\hline & $\mathrm{FI}$ & No & $12.86 \mathrm{~g}$ & $4.92 \mathrm{f}$ & $7.94 \mathrm{~g}$ & 38.3 & 61.70 \\
\hline & & N162 & $17.83 \mathrm{e}$ & $6.56 \mathrm{~d}$ & $11.27 \mathrm{e}$ & 36.8 & 63.20 \\
\hline & & N216 & $18.80 \mathrm{de}$ & $6.85 \mathrm{~cd}$ & $\begin{array}{l}11.95 \\
\text { de }\end{array}$ & 36.45 & 63.55 \\
\hline & & N270 & $19.76 \mathrm{~cd}$ & $7.22 \mathrm{bc}$ & $\begin{array}{l}12.55 \\
\mathrm{~cd}\end{array}$ & 36.53 & 63.47 \\
\hline
\end{tabular}


Note: Different letters in a row are significant differences among treatments in each year $(\mathrm{P}<0.05)$. DI: drip irrigation, SI: micro-sprinkler irrigation, Fl: flooding irrigation. N0: $0 \mathrm{~kg} \mathrm{~N} \mathrm{ha}^{-1}, \mathrm{~N} 162: 162 \mathrm{~kg} \mathrm{~N} \mathrm{ha}^{-1}, \mathrm{~N} 216: 216 \mathrm{~kg} \mathrm{~N} \mathrm{ha}{ }^{-1}, \mathrm{~N} 270$ : 270 kg N ha-1. R6: physiological maturity.

Table 3 Effects of different irrigation methods and nitrogen application rates on nitrogen accumulation and distribution in summer maize. 


\begin{tabular}{|c|c|c|c|c|c|c|c|c|c|}
\hline \multirow[t]{2}{*}{ Year } & \multirow[t]{2}{*}{$\begin{array}{l}\text { Irrigation } \\
\text { method }\end{array}$} & \multirow{2}{*}{$\begin{array}{l}\text { Nitrogen } \\
\text { application rate } \\
\left(\mathrm{kg} \mathrm{ha}^{-1}\right)\end{array}$} & \multicolumn{3}{|c|}{$\begin{array}{l}\text { Nitrogen accumulation } \\
\text { amount of } \mathrm{VT}\left(\mathrm{kg} \mathrm{ha}^{-1}\right)\end{array}$} & \multicolumn{4}{|c|}{$\begin{array}{l}\text { Nitrogen accumulation amount of } \\
\mathrm{R} 6\left(\mathrm{~kg} \mathrm{ha}^{-1}\right)\end{array}$} \\
\hline & & & Stem & Leaf & Plant & Stem & Leaf & Grain & Plant \\
\hline \multirow[t]{12}{*}{2017} & DI & NO & $\begin{array}{l}39.94 \\
f\end{array}$ & $\begin{array}{l}59.89 \\
\text { fgh }\end{array}$ & $99.83 \mathrm{e}$ & $\begin{array}{l}21.80 \\
\mathrm{~g}\end{array}$ & $e^{28.85}$ & $\begin{array}{l}101.44 \\
\mathrm{~g}\end{array}$ & $\begin{array}{l}152.09 \\
\mathrm{~g}\end{array}$ \\
\hline & & N162 & $\begin{array}{l}51.20 \\
\text { cde }\end{array}$ & $\begin{array}{l}64.33 \\
\text { def }\end{array}$ & $\begin{array}{l}115.53 \\
\text { cd }\end{array}$ & $\mathrm{e}^{29.84}$ & $\begin{array}{l}40.42 \\
\mathrm{~cd}\end{array}$ & $\begin{array}{l}142.70 \\
\mathrm{e}\end{array}$ & $\begin{array}{l}212.96 \\
\mathrm{e}\end{array}$ \\
\hline & & N216 & $\begin{array}{l}54.72 \\
\text { abcd }\end{array}$ & $\begin{array}{l}66.65 \\
\text { cde }\end{array}$ & $\begin{array}{l}121.37 \\
a b c\end{array}$ & $\begin{array}{l}33.98 \\
\text { bcd }\end{array}$ & $\begin{array}{l}43.17 \\
\mathrm{bc}\end{array}$ & $\begin{array}{l}170.39 \\
a b\end{array}$ & $\begin{array}{l}247.54 \\
b\end{array}$ \\
\hline & & N270 & $\begin{array}{l}56.28 \\
a b\end{array}$ & $\begin{array}{l}72.32 \\
a b\end{array}$ & $\begin{array}{l}128.60 \\
a\end{array}$ & $\begin{array}{l}37.97 \\
a\end{array}$ & $\begin{array}{l}44.82 \\
a b\end{array}$ & $\begin{array}{l}175.53 \\
a\end{array}$ & $\begin{array}{l}258.31 \\
a\end{array}$ \\
\hline & SI & NO & $\begin{array}{l}41.51 \\
f\end{array}$ & $\begin{array}{l}58.01 \\
\text { gh }\end{array}$ & $99.52 \mathrm{e}$ & $\begin{array}{l}22.45 \\
\mathrm{fg}\end{array}$ & $\mathrm{e}^{29.04}$ & $\begin{array}{l}98.73 \\
\mathrm{~g}\end{array}$ & $\begin{array}{l}150.22 \\
\mathrm{~g}\end{array}$ \\
\hline & & N162 & $\begin{array}{l}51.53 \\
\text { bcde }\end{array}$ & $\begin{array}{l}64.92 \\
\text { def }\end{array}$ & $\begin{array}{l}116.44 \\
\text { bcd }\end{array}$ & $\begin{array}{l}31.82 \\
\mathrm{de}\end{array}$ & $\begin{array}{l}38.68 \\
d\end{array}$ & $\begin{array}{l}144.45 \\
\text { de }\end{array}$ & $\begin{array}{l}214.95 \\
\text { de }\end{array}$ \\
\hline & & N216 & $\begin{array}{l}55.35 \\
\text { abc }\end{array}$ & $\begin{array}{l}66.94 \\
\text { bcde }\end{array}$ & $\begin{array}{l}122.29 \\
a b c\end{array}$ & $\begin{array}{l}32.82 \\
\mathrm{~cd}\end{array}$ & $\begin{array}{l}45.18 \\
a b\end{array}$ & $\begin{array}{l}161.96 \\
\mathrm{C}\end{array}$ & $\begin{array}{l}239.96 \\
C\end{array}$ \\
\hline & & N270 & $\begin{array}{l}57.58 \\
a\end{array}$ & $\begin{array}{l}68.82 \\
\text { abcd }\end{array}$ & $\begin{array}{l}126.40 \\
a\end{array}$ & $\begin{array}{l}36.57 \\
a b\end{array}$ & $\begin{array}{l}47.40 \\
a\end{array}$ & $\begin{array}{l}164.30 \\
\text { bc }\end{array}$ & $\begin{array}{l}248.27 \\
\text { b }\end{array}$ \\
\hline & $\mathrm{FI}$ & NO & $\begin{array}{l}38.54 \\
f\end{array}$ & $\begin{array}{l}56.26 \\
h\end{array}$ & $94.80 \mathrm{e}$ & $\begin{array}{l}24.54 \\
f\end{array}$ & $\begin{array}{l}27.21 \\
\mathrm{e}\end{array}$ & $\begin{array}{l}96.38 \\
\mathrm{~g}\end{array}$ & $\begin{array}{l}148.13 \\
\mathrm{~g}\end{array}$ \\
\hline & & N162 & $\begin{array}{l}47.70 \\
e\end{array}$ & $\begin{array}{l}62.82 \\
\text { efg }\end{array}$ & $\begin{array}{l}110.52 \\
d\end{array}$ & $\begin{array}{l}33.56 \\
\mathrm{~cd}\end{array}$ & $\begin{array}{l}39.20 \\
\mathrm{~d}\end{array}$ & $\begin{array}{l}128.01 \\
f\end{array}$ & $\begin{array}{l}200.77 \\
f\end{array}$ \\
\hline & & N216 & $\begin{array}{l}50.39 \\
\text { de }\end{array}$ & $\begin{array}{l}71.03 \\
a b c\end{array}$ & $\begin{array}{l}121.42 \\
a b c\end{array}$ & $\begin{array}{l}35.15 \\
\text { bc }\end{array}$ & $\begin{array}{l}40.58 \\
\mathrm{~cd}\end{array}$ & $\begin{array}{l}145.90 \\
\text { de }\end{array}$ & $\begin{array}{l}221.62 \\
d\end{array}$ \\
\hline & & N270 & $\begin{array}{l}51.51 \\
\text { bcde }\end{array}$ & $\begin{array}{l}73.68 \\
a\end{array}$ & $\begin{array}{l}125.18 \\
a b\end{array}$ & $\begin{array}{l}38.97 \\
a\end{array}$ & $\begin{array}{l}45.04 \\
a b\end{array}$ & $\begin{array}{l}150.12 \\
d\end{array}$ & $\begin{array}{l}234.14 \\
\mathrm{C}\end{array}$ \\
\hline \multirow[t]{8}{*}{2018} & DI & NO & $\begin{array}{l}27.89 \\
\mathrm{e}\end{array}$ & $\mathrm{e}^{46.11}$ & $74.00 \mathrm{e}$ & $\begin{array}{l}16.86 \\
g\end{array}$ & $\begin{array}{l}20.91 \\
\mathrm{C}\end{array}$ & $\mathrm{e}^{76.96}$ & $\begin{array}{l}114.73 \\
\mathrm{f}\end{array}$ \\
\hline & & N162 & $\begin{array}{l}46.20 \\
\mathrm{~cd}\end{array}$ & $\begin{array}{l}58.29 \\
\mathrm{~cd}\end{array}$ & $\begin{array}{l}104.48 \\
\text { cd }\end{array}$ & $\begin{array}{l}24.68 \\
\text { ef }\end{array}$ & $\begin{array}{l}30.63 \\
b\end{array}$ & $\begin{array}{l}129.60 \\
b c\end{array}$ & $\begin{array}{l}184.91 \\
\mathrm{~d}\end{array}$ \\
\hline & & N216 & $\begin{array}{l}46.38 \\
\text { cd }\end{array}$ & $\begin{array}{l}64.78 \\
a b c\end{array}$ & $\begin{array}{l}111.15 \\
\text { abc }\end{array}$ & $\begin{array}{l}26.43 \\
\mathrm{de}\end{array}$ & $\begin{array}{l}35.92 \\
a\end{array}$ & $\begin{array}{l}158.87 \\
a\end{array}$ & $\begin{array}{l}221.22 \\
a b\end{array}$ \\
\hline & & N270 & $\begin{array}{l}49.12 \\
\text { bc }\end{array}$ & $\begin{array}{l}67.42 \\
a b\end{array}$ & $\begin{array}{l}116.54 \\
a\end{array}$ & $\begin{array}{l}29.79 \\
a b\end{array}$ & $\begin{array}{l}38.20 \\
a\end{array}$ & $\begin{array}{l}160.00 \\
a\end{array}$ & $\begin{array}{l}227.99 \\
a\end{array}$ \\
\hline & SI & NO & $\begin{array}{l}25.26 \\
\text { ef }\end{array}$ & $\begin{array}{l}40.91 \\
\text { ef }\end{array}$ & $\begin{array}{l}66.17 \\
\text { ef }\end{array}$ & $\begin{array}{l}16.54 \\
\mathrm{~g}\end{array}$ & $\begin{array}{l}17.32 \\
d\end{array}$ & $\mathrm{e}^{78.15}$ & $\begin{array}{l}112.01 \\
\mathrm{f}\end{array}$ \\
\hline & & N162 & $\begin{array}{l}43.98 \\
d\end{array}$ & $\begin{array}{l}54.92 \\
\mathrm{~d}\end{array}$ & $98.90 \mathrm{~d}$ & $\begin{array}{l}22.38 \\
f\end{array}$ & $\begin{array}{l}32.37 \\
b\end{array}$ & $\begin{array}{l}131.55 \\
\text { bc }\end{array}$ & $\begin{array}{l}186.30 \\
d\end{array}$ \\
\hline & & N216 & $\begin{array}{l}51.21 \\
a b\end{array}$ & $\begin{array}{l}61.13 \\
\text { bcd }\end{array}$ & $\begin{array}{l}112.34 \\
\text { abc }\end{array}$ & $\begin{array}{l}24.92 \\
\mathrm{de}\end{array}$ & $\begin{array}{l}36.00 \\
a\end{array}$ & $\begin{array}{l}151.58 \\
a\end{array}$ & $\begin{array}{l}212.51 \\
\text { bc }\end{array}$ \\
\hline & & N270 & $\begin{array}{l}54.09 \\
a\end{array}$ & $\begin{array}{l}61.12 \\
\text { bcd }\end{array}$ & $\begin{array}{l}115.21 \\
a b\end{array}$ & $\begin{array}{l}27.25 \\
\mathrm{~cd}\end{array}$ & $\begin{array}{l}37.92 \\
a\end{array}$ & $\begin{array}{l}157.15 \\
a\end{array}$ & $\begin{array}{l}222.31 \\
a b\end{array}$ \\
\hline
\end{tabular}




\begin{tabular}{|c|c|c|c|c|c|c|c|c|}
\hline $\mathrm{FI}$ & No & 24.27 & $\begin{array}{l}37.61 \\
f\end{array}$ & $61.88 \mathrm{f}$ & $\begin{array}{l}15.97 \\
g\end{array}$ & $\begin{array}{l}18.83 \\
\text { cd }\end{array}$ & $e^{68.67}$ & $\begin{array}{l}103.48 \\
f\end{array}$ \\
\hline & N162 & $\begin{array}{l}44.56 \\
d\end{array}$ & $\begin{array}{l}57.38 \\
d\end{array}$ & $\begin{array}{l}101.95 \\
d\end{array}$ & $\begin{array}{l}29.57 \\
a b c\end{array}$ & $\begin{array}{l}30.77 \\
b\end{array}$ & $\begin{array}{l}111.13 \\
\text { d }\end{array}$ & $\begin{array}{l}171.47 \\
\mathrm{e}\end{array}$ \\
\hline & N216 & $\begin{array}{l}45.17 \\
d\end{array}$ & $\begin{array}{l}61.87 \\
\text { bcd }\end{array}$ & $\begin{array}{l}107.05 \\
\text { bcd }\end{array}$ & $\begin{array}{l}28.99 \\
\text { bc }\end{array}$ & $\begin{array}{l}32.22 \\
b\end{array}$ & $\begin{array}{l}125.61 \\
C\end{array}$ & $\begin{array}{l}186.83 \\
d\end{array}$ \\
\hline & N270 & $\begin{array}{l}43.66 \\
d\end{array}$ & $\begin{array}{l}69.93 \\
a\end{array}$ & $\begin{array}{l}113.59 \\
a b\end{array}$ & $\begin{array}{l}31.62 \\
a\end{array}$ & $\begin{array}{l}38.01 \\
a\end{array}$ & $\begin{array}{l}137.96 \\
\text { b }\end{array}$ & $\begin{array}{l}207.58 \\
C\end{array}$ \\
\hline
\end{tabular}

Note: Different letters in a row are significant differences among treatments in each year $(P<0.05)$. DI: drip irrigation, SI: micro-sprinkler irrigation, Fl: flooding irrigation. N0: 0 kg N ha-1, N162: 162 kg N ha-1 , N216: $216 \mathrm{~kg} \mathrm{~N} \mathrm{ha}^{-1}$, N270: 270 kg N ha ${ }^{-1}$. VT: tasseling, R6: physiological maturity.

Table 4 Nitrogen translocation efficiency and contribution of post-tasseling to grain as affected by the different irrigation methods and nitrogen application rates. 


\begin{tabular}{|c|c|c|c|c|c|c|c|}
\hline Year & $\begin{array}{l}\text { Irrigation } \\
\text { method }\end{array}$ & $\begin{array}{l}\text { Nitrogen } \\
\text { application } \\
\text { rate }(\mathrm{kg} \\
\left.\mathrm{ha}^{-1}\right)\end{array}$ & $\begin{array}{l}\mathrm{N} \\
\text { translocation } \\
\left(\mathrm{kg} \mathrm{ha}^{-1}\right)\end{array}$ & $\begin{array}{l}\mathrm{N} \\
\text { translocation } \\
\text { efficiency } \\
(\%)\end{array}$ & $\begin{array}{l}\text { Contribution } \\
\text { of } \mathrm{N} \\
\text { translocation } \\
\text { to grain (\%) }\end{array}$ & $\begin{array}{l}\mathrm{N} \\
\text { assimilation } \\
\left(\mathrm{kg} \mathrm{ha}^{-1}\right)\end{array}$ & $\begin{array}{l}\text { Contribution } \\
\text { of } \mathrm{N} \\
\text { assimilation } \\
\text { to grain (\%) }\end{array}$ \\
\hline \multirow[t]{12}{*}{2017} & DI & NO & $49.18 \mathrm{a}$ & $48.96 \mathrm{a}$ & $48.51 \mathrm{a}$ & $52.26 \mathrm{e}$ & $51.49 \mathrm{~b}$ \\
\hline & & N162 & $45.27 \mathrm{ab}$ & $39.16 \mathrm{bcd}$ & $31.76 \mathrm{~b}$ & $97.43 \mathrm{~cd}$ & $68.24 \mathrm{a}$ \\
\hline & & N216 & $44.22 \mathrm{ab}$ & $36.34 \mathrm{~cd}$ & $25.93 \mathrm{~b}$ & $126.17 \mathrm{a}$ & $74.07 \mathrm{a}$ \\
\hline & & N270 & $45.82 \mathrm{ab}$ & $35.63 \mathrm{~cd}$ & $26.11 \mathrm{~b}$ & $129.71 \mathrm{a}$ & $73.89 a$ \\
\hline & SI & No & $43.05 a b$ & $45.32 \mathrm{ab}$ & $44.65 \mathrm{a}$ & $53.33 \mathrm{e}$ & $55.35 \mathrm{~b}$ \\
\hline & & N162 & $37.76 \mathrm{~b}$ & $34.06 \mathrm{~cd}$ & $29.63 \mathrm{~b}$ & $90.25 \mathrm{~d}$ & $70.37 a$ \\
\hline & & N216 & $45.70 \mathrm{ab}$ & $37.48 \mathrm{~cd}$ & $31.30 \mathrm{~b}$ & $100.20 \mathrm{~cd}$ & $68.70 \mathrm{a}$ \\
\hline & & N270 & $41.17 \mathrm{ab}$ & $32.85 \mathrm{~d}$ & $27.41 \mathrm{~b}$ & 108.96 bc & 72.59 a \\
\hline & $\mathrm{FI}$ & NO & $48.03 \mathrm{ab}$ & $48.11 \mathrm{a}$ & $48.60 \mathrm{a}$ & $50.70 \mathrm{e}$ & $51.40 \mathrm{~b}$ \\
\hline & & N162 & $45.94 \mathrm{ab}$ & $39.36 \mathrm{bc}$ & $31.87 \mathrm{~b}$ & $98.51 \mathrm{~cd}$ & $68.13 \mathrm{a}$ \\
\hline & & N216 & $44.30 \mathrm{ab}$ & $36.19 \mathrm{~cd}$ & $27.40 \mathrm{~b}$ & $117.66 \mathrm{ab}$ & $72.60 \mathrm{a}$ \\
\hline & & N270 & $42.43 a b$ & $33.53 \mathrm{~cd}$ & $25.85 b$ & $121.87 \mathrm{a}$ & $74.15 \mathrm{a}$ \\
\hline \multirow[t]{12}{*}{2018} & DI & No & $36.23 \mathrm{~cd}$ & $48.98 \mathrm{a}$ & $47.08 \mathrm{a}$ & $40.73 \mathrm{f}$ & $52.92 \mathrm{f}$ \\
\hline & & N162 & $49.17 \mathrm{ab}$ & $47.06 \mathrm{ab}$ & $37.96 \mathrm{bcd}$ & $80.43 \mathrm{de}$ & $62.04 \mathrm{cde}$ \\
\hline & & N216 & $48.80 \mathrm{ab}$ & $43.75 \mathrm{abcd}$ & 30.91 ef & 110.07 a & $69.09 a b$ \\
\hline & & N270 & $48.55 a b$ & $41.42 \mathrm{~cd}$ & $30.52 \mathrm{f}$ & $111.45 \mathrm{a}$ & $69.48 \mathrm{a}$ \\
\hline & SI & NO & $27.08 \mathrm{e}$ & $43.69 \mathrm{abcd}$ & $39.50 \mathrm{bc}$ & $41.60 \mathrm{f}$ & $60.50 \mathrm{de}$ \\
\hline & & N162 & $41.60 \mathrm{bc}$ & $40.80 \mathrm{~cd}$ & 37.56 bcde & $69.52 \mathrm{e}$ & 62.44 bcde \\
\hline & & N216 & $45.83 \mathrm{ab}$ & $42.81 \mathrm{bcd}$ & 36.49 bcdef & $79.79 \mathrm{de}$ & 63.51 abcde \\
\hline & & N270 & $43.97 \mathrm{abc}$ & $38.67 \mathrm{~d}$ & 31.86 def & $93.99 \mathrm{bc}$ & $68.14 a b c$ \\
\hline & $\mathrm{FI}$ & NO & $32.31 \mathrm{de}$ & 48.79 a & $41.32 \mathrm{ab}$ & $45.84 \mathrm{f}$ & 58.68 ef \\
\hline & & N162 & $44.15 \mathrm{abc}$ & $44.53 \mathrm{abc}$ & $33.51 \mathrm{cdef}$ & $87.40 \mathrm{~cd}$ & $66.49 \mathrm{abcd}$ \\
\hline & & N216 & $51.42 \mathrm{a}$ & $45.74 \mathrm{abc}$ & 33.87 cdef & $100.16 \mathrm{abc}$ & $66.13 \mathrm{abcd}$ \\
\hline & & N270 & $50.05 \mathrm{a}$ & $43.46 \mathrm{abcd}$ & 31.88 def & $107.10 \mathrm{ab}$ & $68.12 a b c$ \\
\hline
\end{tabular}

Note: Different letters in a row are significant differences among treatments in each year $(\mathrm{P}<0.05)$. DI: drip irrigation, SI: micro-sprinkler irrigation, Fl: flooding irrigation. $\mathrm{N0}: 0 \mathrm{~kg} \mathrm{~N} \mathrm{ha}^{-1}, \mathrm{~N} 162: 162 \mathrm{~kg} \mathrm{~N} \mathrm{ha}^{-1}, \mathrm{~N} 216: 216 \mathrm{~kg} \mathrm{~N} \mathrm{ha}^{-1}$, N270: $270 \mathrm{~kg} \mathrm{~N} \mathrm{ha}^{-1}$.

Table 5 Effects of different irrigation methods and nitrogen application rates on the nitrogen use efficiency indicators in summer maize. 


\begin{tabular}{|c|c|c|c|c|c|c|}
\hline Year & $\begin{array}{l}\text { Irrigation } \\
\text { method }\end{array}$ & $\begin{array}{l}\text { Nitrogen application rate }(\mathrm{kg} \\
\left.\mathrm{ha}^{-1}\right)\end{array}$ & $\mathrm{NHI}(\%)$ & NUE (\%) & $\begin{array}{l}\mathrm{AE}_{\mathrm{N}}\left(\mathrm{kg} \mathrm{kg}^{-}\right. \\
1)\end{array}$ & $\begin{array}{l}\mathrm{PFP}_{\mathrm{N}}\left(\mathrm{kg} \mathrm{kg}^{-}\right. \\
\left.{ }^{1}\right)\end{array}$ \\
\hline \multirow[t]{12}{*}{2017} & DI & NO & $\begin{array}{l}66.73 \\
a b c\end{array}$ & - & - & - \\
\hline & & N162 & $\begin{array}{l}67.01 \\
\text { abc }\end{array}$ & $\begin{array}{l}38.28 \\
\mathrm{bcd}\end{array}$ & $25.95 \mathrm{bcd}$ & 73.58 a \\
\hline & & N216 & $68.83 a$ & $45.02 \mathrm{a}$ & $32.52 \mathrm{a}$ & $58.10 \mathrm{c}$ \\
\hline & & N270 & $67.96 \mathrm{ab}$ & $\begin{array}{l}40.08 \\
\mathrm{bc}\end{array}$ & $27.96 \mathrm{bc}$ & $47.46 \mathrm{e}$ \\
\hline & SI & NO & $\begin{array}{l}65.71 \\
\text { bcde }\end{array}$ & - & - & - \\
\hline & & N162 & $\begin{array}{l}67.20 \\
a b c\end{array}$ & $\begin{array}{l}40.71 \\
\mathrm{bc}\end{array}$ & $28.76 a b$ & $71.79 a$ \\
\hline & & N216 & $67.49 a b$ & $\begin{array}{l}42.33 \\
a b\end{array}$ & $29.83 a b$ & $57.59 \mathrm{c}$ \\
\hline & & N270 & $\begin{array}{l}66.18 \\
\text { bcd }\end{array}$ & $\begin{array}{l}37.00 \\
\text { cde }\end{array}$ & $24.74 \mathrm{~cd}$ & $46.42 \mathrm{e}$ \\
\hline & $\mathrm{FI}$ & NO & $\begin{array}{l}65.08 \\
\text { cde }\end{array}$ & - & - & - \\
\hline & & N162 & $63.76 \mathrm{e}$ & 33.11 ef & $19.89 \mathrm{e}$ & $69.09 \mathrm{~b}$ \\
\hline & & N216 & $\begin{array}{l}65.79 \\
\text { bcde }\end{array}$ & $\begin{array}{l}34.67 \\
\text { def }\end{array}$ & $23.36 \mathrm{de}$ & $54.09 \mathrm{~d}$ \\
\hline & & N270 & $64.12 \mathrm{de}$ & $32.46 f$ & $20.28 \mathrm{e}$ & $45.89 \mathrm{e}$ \\
\hline \multirow[t]{11}{*}{2018} & $\mathrm{DI}$ & No & $67.16 \mathrm{~b}$ & - & - & - \\
\hline & & N162 & $70.09 a$ & $\begin{array}{l}44.14 \\
a b\end{array}$ & $33.11 \mathrm{ab}$ & $63.95 \mathrm{a}$ \\
\hline & & N216 & $71.80 \mathrm{a}$ & $50.23 \mathrm{a}$ & $38.63 \mathrm{a}$ & $52.67 \mathrm{c}$ \\
\hline & & N270 & $70.16 \mathrm{a}$ & $\begin{array}{l}42.74 \\
a b\end{array}$ & 31.34 bc & $43.51 \mathrm{e}$ \\
\hline & SI & NO & $69.79 a$ & - & - & - \\
\hline & & N162 & $70.63 \mathrm{a}$ & $\begin{array}{l}46.73 \\
a b\end{array}$ & $33.59 a b$ & $65.22 \mathrm{a}$ \\
\hline & & N216 & $71.34 \mathrm{a}$ & $\begin{array}{l}47.40 \\
a b\end{array}$ & $34.64 a b$ & $52.32 \mathrm{c}$ \\
\hline & & N270 & 70.68 a & $\begin{array}{l}41.62 \\
a b\end{array}$ & $29.81 b c$ & $43.38 \mathrm{e}$ \\
\hline & $\mathrm{FI}$ & NO & $66.37 \mathrm{bc}$ & - & - & - \\
\hline & & N162 & $64.81 \mathrm{c}$ & $\begin{array}{l}42.76 \\
a b\end{array}$ & $26.70 \mathrm{c}$ & $57.18 \mathrm{~b}$ \\
\hline & & N216 & $67.23 \mathrm{~b}$ & $39.32 \mathrm{~b}$ & $26.86 \mathrm{c}$ & $49.88 \mathrm{~d}$ \\
\hline
\end{tabular}


Note: Different letters in a row are significant differences among treatments in each year $(\mathrm{P}<0.05)$. DI: drip irrigation, SI: micro-sprinkler irrigation, Fl: flooding irrigation. N0: $0 \mathrm{~kg} \mathrm{~N} \mathrm{ha}^{-1}, \mathrm{~N} 162: 162 \mathrm{~kg} \mathrm{~N} \mathrm{ha}^{-1}, \mathrm{~N} 216: 216 \mathrm{~kg} \mathrm{~N} \mathrm{ha}^{-1}, \mathrm{~N} 270$ : $270 \mathrm{~kg} \mathrm{~N} \mathrm{ha}{ }^{-1}$. $A E_{N}$ : agronomic efficiency of applied $\mathrm{N}, \mathrm{NHI}: \mathrm{N}$ harvest index, NUE: $\mathrm{N}$ use efficiency, $\mathrm{PFP}_{\mathrm{N}}: \mathrm{N}_{\text {partial }}$ factor productivity.

\section{Figures}

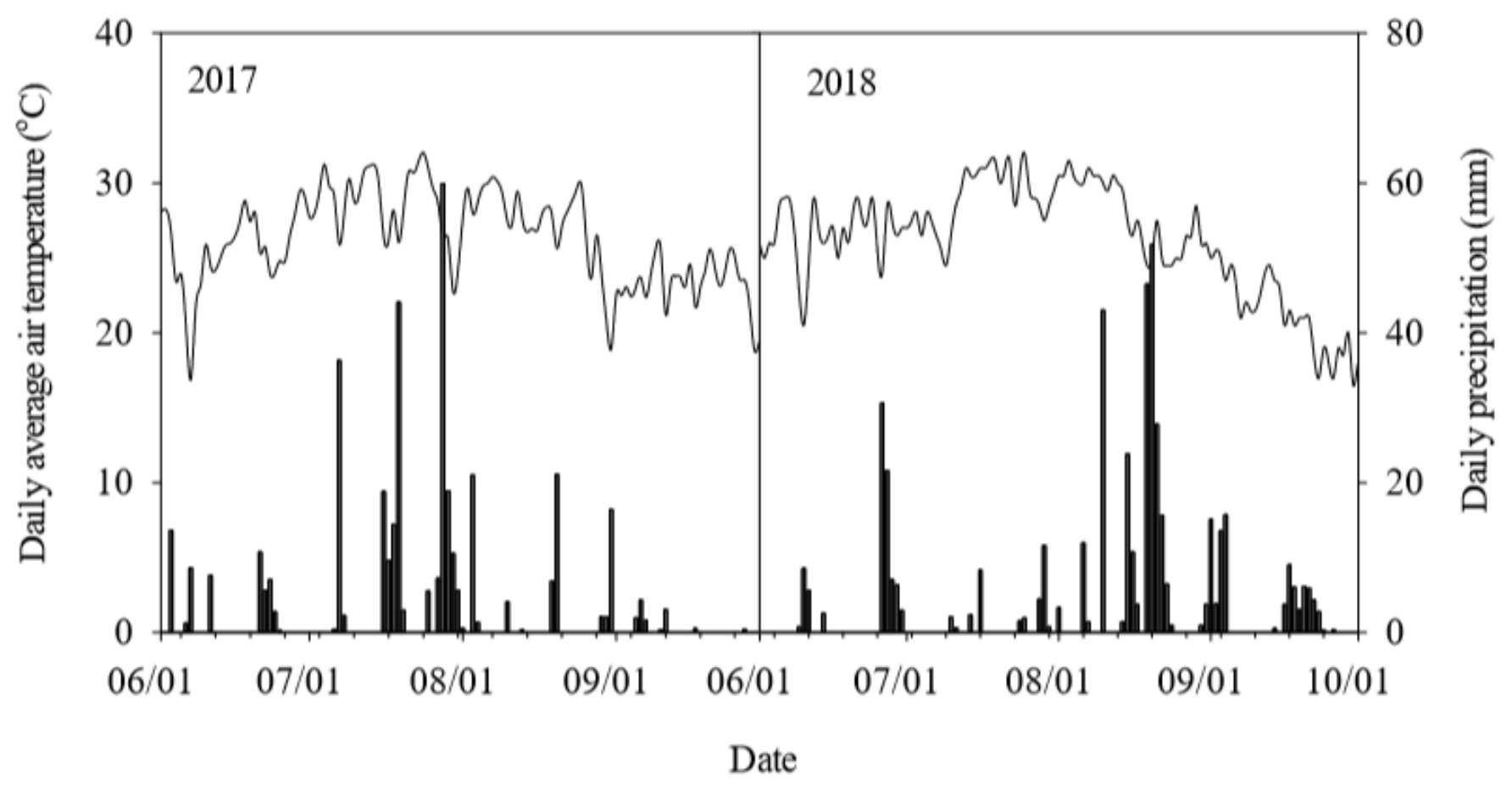

Figure 1

The daily average air temperature and precipitation of the growth stages for summer maize in 2017 and 2018. 

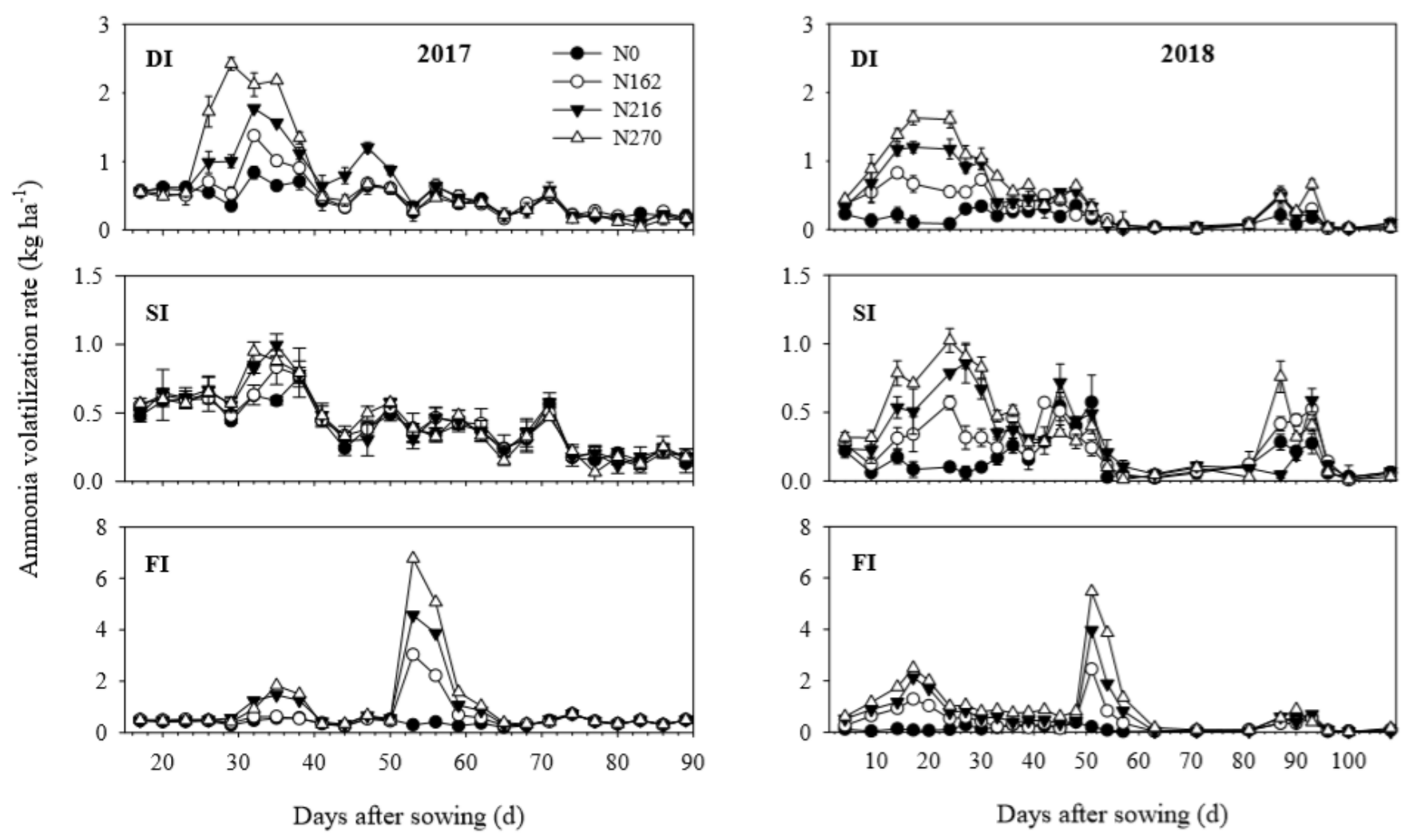

Figure 2

Effects of different irrigation methods and nitrogen application rates on ammonia volatilization in 2017 and 2018 . DI: drip irrigation, SI: micro-sprinkler irrigation, Fl: flooding irrigation. N0: 0 kg N ha-1, N162: 162 kg N ha-1, N216: 216 kg N ha-1, N270: 270 kg N ha-1 


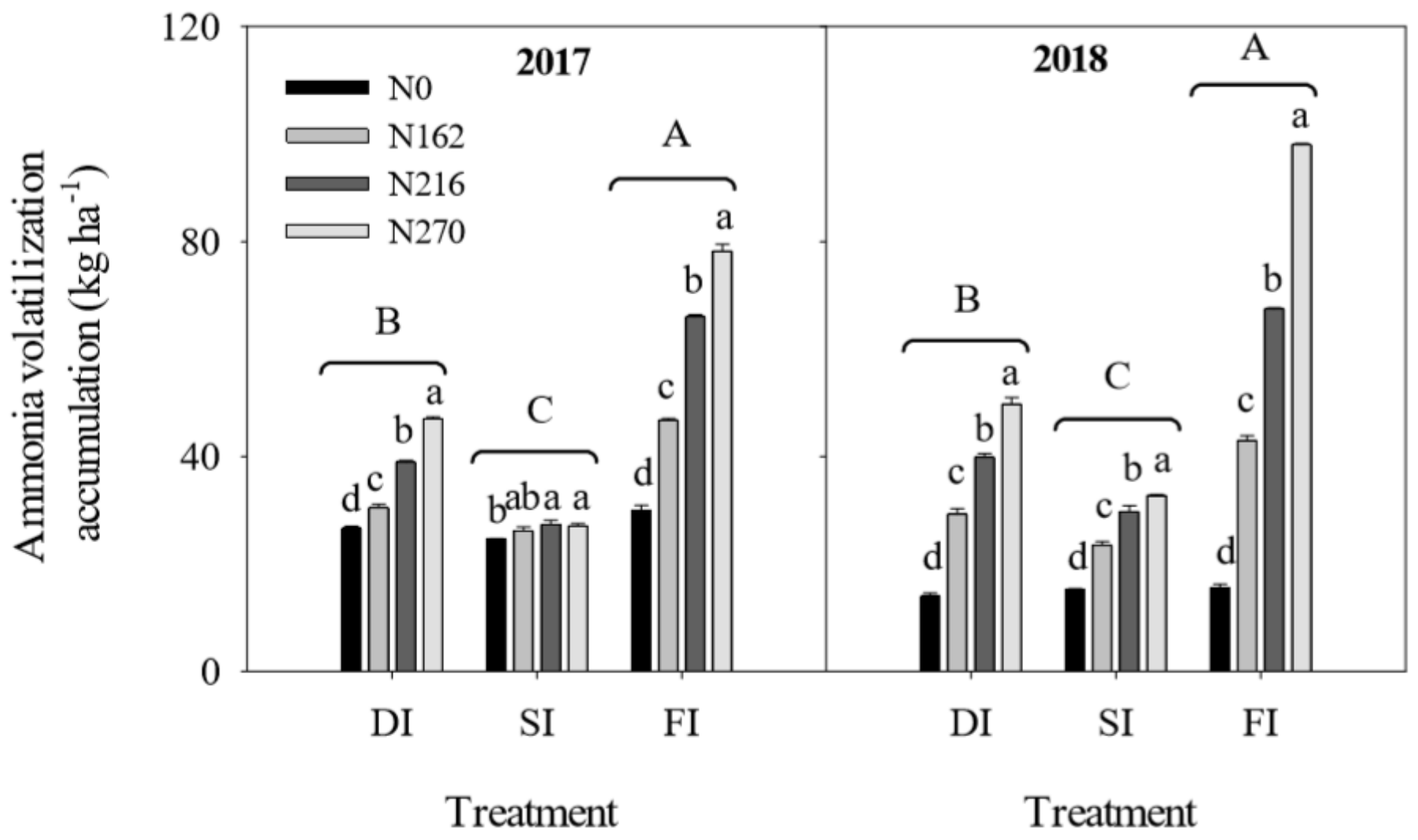

Figure 3

Effects of different irrigation methods and nitrogen application rates on ammonia volatilization accumulation of soil. Different capital or small values are significant differences among treatments in each $\mathrm{N}$ application rate at the $5 \%$ level. DI: Drip irrigation, SI: Micro-sprinkler irrigation, Fl: Flooding irrigation. N0: 0 kg N ha-1, N162: 162 kg N ha-1, N216: 216 kg N ha-1, N270: 270 kg N ha-1. 


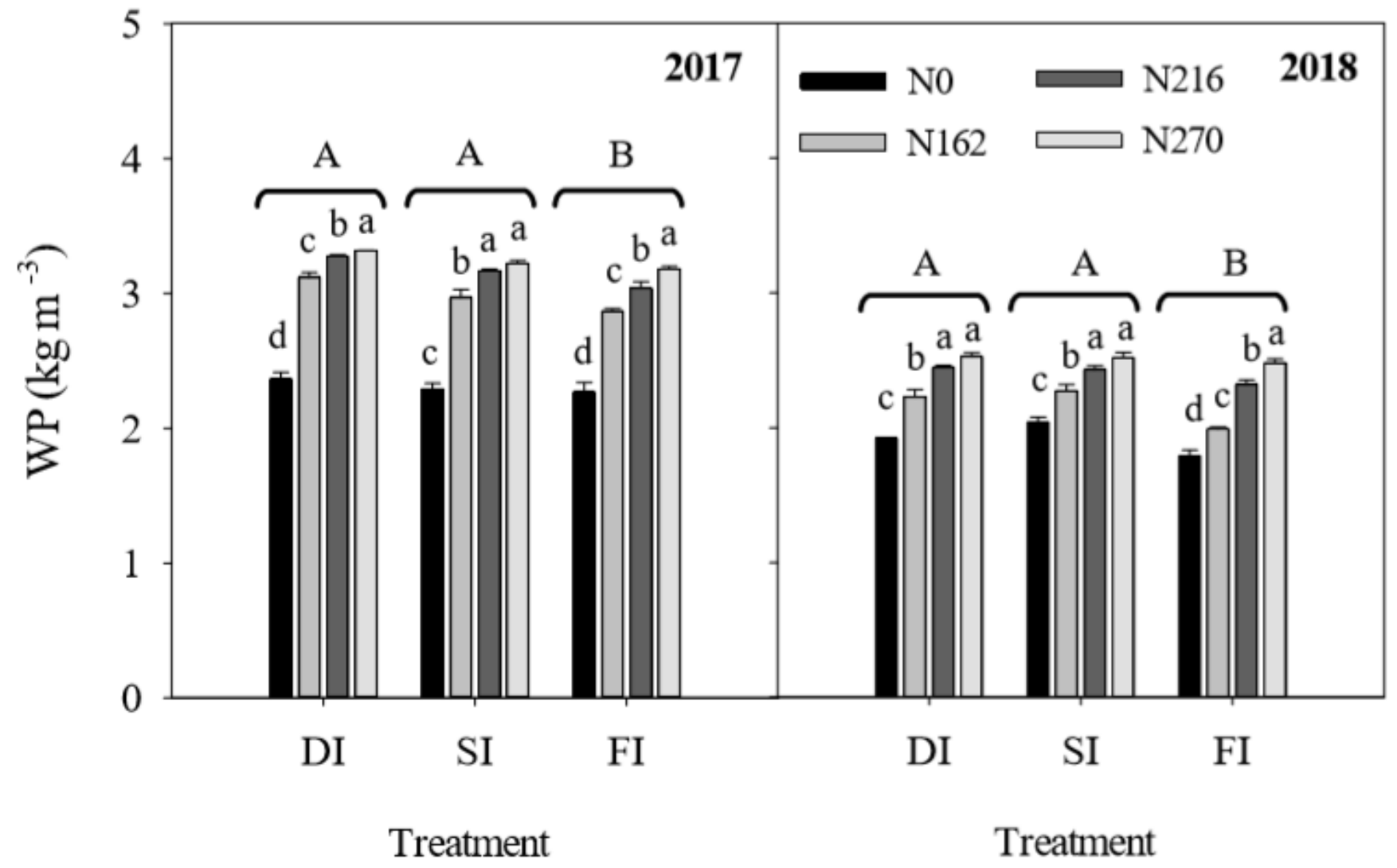

Figure 4

Effects of different irrigation methods and nitrogen application rates on water productivity (WP) in summer maize. Different capital or small values are significant differences among treatments in each $\mathrm{N}$ application rate at the $5 \%$ level. DI: drip irrigation, SI: micro-sprinkler irrigation, Fl: flooding irrigation. N0: 0 kg N ha -1, N162: 162 kg N ha -1, N216: 216 kg N ha -1, N270: 270 kg N ha -1. 